\title{
Identification of 4-Aminopyrazolopyrimidine Metabolite That May Contribute to the Hypolipidemic Effects of LY2584702 in Long Evans Diet-Induced Obese Rats ${ }^{\mathbf{S}}$
}

\author{
Thomas B. Estridge, Asim B. Dey, Charles Reidy, Xiaohong Yu, Yuke Zhang, \\ Maryalice Hartley, Paul L. Milligan, Najia Jin, Mark C. Kowala, Jennifer K. Leohr, \\ Adrian J. Fretland, Thomas E. Mabry, Debra Luffer-Atlas, and M. Jane Luo \\ CardioMet Disease/Diabetic Complications (T.B.E., A.B.D., C.R., X.Y., Y.Z., N.J., M.H., M.C.K, M.J.L.), Discovery Chemistry and \\ Research Technologies (P.L.M., T.E.M.), Global PK/PD \& Pharmacometrics (J.K.L.), Drug Disposition (A.J.F., D.L.-A.), Eli Lilly \& \\ Co., Indianapolis, Indiana
}

Received January 13, 2017; accepted April 26, 2017

\begin{abstract}
LY2584702 is an inhibitor of p70 S6 kinase-1 previously developed for the treatment of cancer. In two phase 1 trials in oncology patients, significant reductions of total cholesterol, low-density lipoprotein cholesterol (LDL-C), and triglyceride were observed. In the current study, we sought to understand the potential mechanism of action of this compound in regulating lipid metabolism. In Long Evans diet-induced obese (DIO) rats, oral administration of LY2584702 for 3-4 weeks led to robust reduction of LDL-C up to $60 \%$. An unexpected finding of liver triglyceride (TG) increase implicated a metabolite of LY2584702, 4-aminopyrazolo[3,4-day]pyrimidine (4-APP), in modulation of lipid metabolism in these rats. We showed that low-dose 4-APP,
\end{abstract}

when administered orally for 3-4 weeks to Long Evans DIO rats, produced lipoprotein profile changes that were strikingly similar to LY2584702. Kinetic studies suggested that both LY2584702 and 4-APP had no effect on chylomicron-TG secretion and only exerted a modest effect on hepatic very low-density lipoprotein (VLDL)-TG secretion. In human hepatoma HepG2 cells, 4-APP, but not LY2584702, increased LDL uptake. We hypothesize that generation of the 4-APP metabolite may contribute to the efficacy of LY2584702 in lowering LDL-C in rats and potentially in humans as well. This mechanism of LDL-C lowering may include inhibition of VLDL production and increase in LDL clearance.

\section{Introduction}

P70 S6 kinase-1 (p70S6K1) is a serine/threonine protein kinase downstream of the phosphatidylinositol 3 kinase/ protein kinase $\mathrm{B} / \mathrm{mammalian}$ target of rapamycin signaling pathway (Pearce et al., 2010; Magnuson et al., 2012). Studies in the past decade suggest p70S6K1 is an important regulator of lipid and energy metabolism (Ricoult and Manning, 2013). The p70S6K1 pathway promotes processing and activation of sterol regulatory element-binding protein 1c (SREBP1c), a transcription factor that regulates expression of genes involved in de novo fatty acid synthesis, de novo sterol synthesis, as well as lipoprotein metabolism (Owen et al., 2012). Knockdown of hepatic S6K1 in db/db mice resulted in downregulation of SREBP1c-responsive genes in the liver as well as reduced

https://doi.org/10.1124/jpet.117.240242.

S This article has supplemental material available at jpet.aspetjournals.org. hepatic triglyceride content and serum triglyceride concentration (Li et al., 2011).

LY2584702 tosylate (hereafter referred to as LY2584702) is a selective inhibitor of p70S6K1 previously developed for treatment of cancer (Hollebecque et al., 2014; Tolcher et al., 2014). In two independent clinical trials in cancer patients, reductions in serum total cholesterol and triglyceride were observed after 2-6 weeks of treatment (Hollebecque et al., 2014; Tolcher et al., 2014). Many patients exhibited greater than $50 \%$ reduction in total cholesterol from baseline. When serum samples were fractionated, both low-density lipoprotein cholesterol (LDL-C) and high-density lipoprotein cholesterol (HDL-C) were found to be reduced, with LDL-C reduced to a greater extent (Tolcher et al., 2014). These changes in serum lipids associated with LY2584702 treatment were directionally consistent with the postulated role of p70S6K1 in regulation of lipid metabolism. However, the robustness of

ABBREVIATIONS: ACC1, acetyl-CoA carboxylase 1; ALT, alanine aminotransferase; 4-APP, 4-aminopyrazolo[3,4-d]pyrimidine; ACLY, ATP citrate lyase; CE, cholesterol ester; Cyp7a1, cytochrome P450 family 7 subfamily A member 1 ; DPBS, Dulbecco's phosphate-buffered saline; FASN, fatty acid synthase; HDL, high-density lipoprotein; HDL-C, HDL cholesterol; HMGCR, 3-hydroxy-3-methyl-glutaryl-coenzyme A reductase; HPLC, highperformance LC; LC, liquid chromatography; LDL-C, low-density lipoprotein-cholesterol; LDLR, low-density lipoprotein receptor; LE-DIO rats, Long Evans diet-induced obese rats; LPE, lysophosphatidylethanolamine; LY2584702, LY2584702 tosylate; mpk, milligram per kilogram; MTP, microsomal triglyceride transfer protein; MTPi, MTP inhibitor lomitapibe; PE, phosphatidylethanolamine; PCSK9, proprotein convertase subtilisin kexin 9; p70S6K1, P70 S6 kinase-1; PCR, polymerase chain reaction; SCD1, stearoyl-CoA desaturase; SREBP, sterol regulatory element-binding protein; TG, triglyceride; VLDL, very low-density lipoprotein. 
the response also begs the question whether additional mechanism may be involved.

Circulating levels of LDL are balanced by its rate of production and clearance. Several classes of lipid-lowering drugs are known to work by modulating these key pathways. For example, statins inhibit cholesterol biosynthesis. Through feedback regulation of gene expression, statins also enhance LDL clearance via upregulation of LDL receptor (LDLR) mRNA (Goldstein and Brown, 2015). Proprotein convertase subtilisin kexin 9 (PCSK9) inhibitors enhance LDL clearance by increasing cell surface LDLR protein levels (Horton et al., 2009; Seidah et al., 2014). Microsomal transfer protein (MTP) inhibitor lomitapibe blocks production of very low-density lipoprotein (VLDL) and chylomicron (Rader and Kastelein, 2014). In this work, we asked whether LY2584702 perturbs any of these pathways both in vivo in rodent models and in vitro in human hepatoma cell systems.

During the course of these investigations, we identified a metabolite of LY2584702 (4-aminopyrazolo[3,4-day]pyrimidine; 4-APP) that accumulates in the liver of Long Evans diet-induced obese (LE-DIO) rats after oral administration of LY2584702. When administered orally to LE-DIO rats, LY2584702 and low-dose 4-APP led to comparable reductions in serum LDLC. Kinetics studies showed that both LY2584702 and lowdose 4-APP had no effect on chylomicron secretion and only minimal effect on VLDL secretion, suggesting enhanced LDL clearance may play a role. Indeed in a human hepatoma HepG2 cell line, 4-APP enhanced LDL uptake. We hypothesize that formation of the 4-APP metabolite may contribute to the efficacy of LY2584702 in lowering LDL-C in the LE-DIO rat model. This study also highlights the importance of metabolite identification work and emphasizes the need to investigate potential metabolites that may contribute to both efficacy and/or safety of an investigational drug.

\section{Materials and Methods}

Materials. HepG2 cells were obtained from ATCC (cat. no. HB-8065; Manassas, VA). 4-APP (20289-44-5) was obtained from SigmaAldrich (St. Louis, MO). Both unlabeled LY2584702 tosylate and $\left[{ }^{14} \mathrm{C}\right]$ LY2584702 tosylate (specific activity $52.4 \mu \mathrm{Ci} / \mathrm{mg}$ ) were synthesized at Eli Lilly and Company (Indianapolis, IN).

Bioanalytical Methods for LY2584702 and 4-APP Metabolite. The tissue samples were homogenized in $3 \mathrm{ml}$ of methanol/water $(1: 1, v / v)$ for each gram of tissue with a Bio-Gen Pro200 tissue grinder (Pro Scientific, Oxford CT). Calibration standards were prepared by serial dilution of a $100-\mu \mathrm{g} / \mathrm{ml}$ analyte stock solution with methanol/ water $(1: 1, v / v)$ to produce working solutions that were then used to fortify control plasma and tissue homogenates to yield analyte concentrations of $1,2.5,5,25,125,250,1250$, and $5000 \mathrm{ng} / \mathrm{ml}$. A $25-\mu \mathrm{l}$ aliquot of each study sample, appropriate calibration standard, and control matrix were transferred to a 96 -well plate, then mixed with $180 \mu \mathrm{l}$ of acetonitrile/methanol (1:1, v/v) saturated with ascorbic acid containing internal standard. After mixing, the samples were centrifuged to pellet the precipitated proteins, and $50 \mu \mathrm{l}$ of the resulting supernatants were transferred to a clean 96 -well plate, evaporated to dryness, and then reconstituted with $100 \mu \mathrm{l}$ acetonitrile for 4-APP analysis, and aliquots of the original supernatants were analyzed for LY2584702.

4-APP was quantified with a Sciex API6500 triple quadrupole mass spectrometer (Applied Biosystems/MDS, Foster City, CA) equipped with a TurboIonSpray interface, and operated in positive ion mode. The analyte and internal standard were chromatographically separated using an Atlantis HILIC 5- $\mu \mathrm{m} 2.1 \times 50 \mathrm{~mm}$ high-performance liquid chromatography (HPLC) column (Waters Corporation, Milford, MA) and a gradient elution. LC conditions were: water/2 M ammonium bicarbonate (1000:25, v/v) (mobile phase A), and acetonitrile/acetic acid $(1000: 0.5, \mathrm{v} / \mathrm{v})$ (mobile phase B). The gradient profile changed from $99.9 \% \mathrm{~B}$ at 0 minutes, $98 \% \mathrm{~B}$ at $0.01-0.33$ minutes, $84 \% \mathrm{~B}$ at $0.43-0.50$ minutes, and $20 \%$ at $0.51-0.70$ minutes. The flow rate was $1.5 \mathrm{ml} / \mathrm{min}$. Chromatography was performed at ambient temperature, with flow directed to the mass spectrometer between 0.30 and 0.70 minutes. Selected reaction monitoring $(\mathrm{M}+\mathrm{H})^{+} \mathrm{m} / z$ transitions were $136.1>64.0$ for 4 -APP and $150.1>133.1$ for $N$-methyl adenine (internal standard). It is important to note that the 64.0-Da product ion in 4-APP is not detected in the tandem mass spectrum of adenine, which is an isobaric isomer. Thus, it was concluded that measurement of 4-APP concentrations were not skewed by interference with endogenous adenine. The TurboIonSpray temperature was maintained at $500^{\circ} \mathrm{C}$, with collision, curtain, nebulizing, and desolvation gas (nitrogen) settings of 30,45 , and 60 , respectively. The ionspray voltage was set to $5500 \mathrm{~V}$, and the respective declustering, entrance, and exit potentials were 40,10 , and 11 .

LY2584702 was determined with a Sciex API4000 triple quadrupole mass spectrometer (Applied Biosystems/MDS). Analyte and internal standard were separated using a Betasil C18 5- $\mu \mathrm{m} 2.1 \times 20 \mathrm{~mm}$ Javelin (Thermo Electron Companies., Madison, WI) and LC conditions: water/2 M ammonium bicarbonate (1000:2.5, v/v) (mobile phase A), and methanol/2 M ammonium bicarbonate (1000:2.5, v/v) (mobile phase B). The gradient profile changed from $10 \% \mathrm{~B}$ at 0 minutes, $47 \% \mathrm{~B}$ at $0.01-0.20$ minutes, $77 \% \mathrm{~B}$ at $0.30-0.40$ minutes, and $98 \%$ at $0.41-0.72$ minutes. The flow rate was $1.5 \mathrm{ml} / \mathrm{min}$. Chromatography was performed at ambient temperature, with flow directed to the mass spectrometer between 0.25 and 0.50 minutes. The selected reaction monitoring $(\mathrm{M}+\mathrm{H})^{+} \mathrm{m} / z$ transition was $446.2>311.2$. The TurboIonSpray temperature was maintained at $740^{\circ} \mathrm{C}$, with collision, curtain, nebulizing, and desolvation gas (nitrogen) settings of 50, 10, 50, and 70 , respectively. The ionspray voltage was set to $1500 \mathrm{~V}$, and the respective declustering, entrance, and exit potentials were 80,10 , and 9 .

Gene Expression Analysis in HepG2 Assay. The effect of 4-APP and LY2584702 on gene expression was determined using HepG2 cells (ATCC; cat. no. HB-8065; Manassas, VA). Cells were plated at 50,000 cells/well on poly-D-lysine-coated 96-well plates (cat. no. 354461; Corning Life Sciences, Teterboro, NJ) in MEM $\alpha$ Media (Gibco, cat. no. 12561-049; Thermo Fisher Scientific, Waltham, MA)/1\% MEM Essential Vitamins (cat. no. 13-607C; Lonza, Allendale, NJ)/10\% HI FBS (Gibco, cat. no. 10082-147; Thermo Fisher Scientific)/1\% Pen/Strep (Gibco, cat. no. 15140-122; Thermo Fisher Scientific), then incubated overnight at $37^{\circ} \mathrm{C}$ and $5 \% \mathrm{CO}_{2}$ in humidified chambers. Cells were treated with LY2584702 and 4-APP $(N=4)$ at the indicated concentrations for 24 hours at $37^{\circ} \mathrm{C}$ and $5 \% \mathrm{CO}_{2}$ in humidified chambers. Total RNA was isolated using QIAGEN RNeasy Mini Kit (cat. no. 74104; Hilden, Germany). cDNA was synthesized using SuperScript III First-Strand Synthesis System for reverse transcription-polymerase chain reaction (RT-PCR; cat. no. 18080051; Thermo Fisher Scientific). Realtime PCR was carried out using the 7900 HT Sequence Detection System from Applied Biosystems per manufacturer's instructions. All reagents were from ThermoFisher Scientific. These include TaqMan Gene Expression Master Mix (cat. no. 4369016); primer and probe mixed for 18S rRNA (cat. no. 4310893E); human acetyl-CoA carboxylase 1 (ACC1; Hs01046047_m1); human ATP citrate lyase (ACLY) mRNA (Hs00982738_m1); human fatty acid synthase (FASN) mRNA (Hs01005622_m1); human stearoyl-CoA desaturase 1 (SCD1) mRNA (Hs01682761_m1); human PCSK9 mRNA (Hs00545399_m1); human 3-hydroxy-3-methyl-glutaryl-coenzyme A reductase mRNA (HMGCR; Hs01102995_g1); and human LDLR (Hs00181192_m1).

Rat PCSK9 Enzyme-Linked Immunosorbent Assay. Rat PCSK9 levels were determined using an enzyme-linked immunosorbent assay. Specifically, Immulon 4HBX 96-well plates (Thermo Fisher Scientific) were coated with $100 \mu \mathrm{l}$ anti-PCSK9 antibody (diluted 1:100, cat. no. AF3888; R\&D Systems, Minneapolis, MN) overnight at $4^{\circ} \mathrm{C}$. The plate was washed three times with phosphate-buffered saline/0.1\% 
Tween 20 and then blocked with $200 \mu$ l of casein blocking buffer (cat. no. 37528; Pierce, Waltham, MA) for 1 hour at room temperature. The plate was washed three times, then $100-\mu \mathrm{l}$ samples and standards were added to the plate and incubated for 16 hours at room temperature with slow rotation on a plate shaker. The plate was washed three times, then $100 \mu \mathrm{l}$ of biotinylated anti-PCSK9 antibody (cat. no. BAF3888, diluted 1:100; R\&D Systems) was added to the plate and incubated for 2 hours at room temperature with slow rotation on a plate shaker. The plate was washed three times, then $100 \mu \mathrm{l}$ of streptavidin-horseradish peroxidase conjugate (diluted 1:5000, cat. no. 18-152; Millipore, Temecula, CA) was added to the plate and incubated for 1 hour at room temperature with slow rotation on a plate shaker. The plate was washed three times, then $100 \mu \mathrm{l}$ of color reagent (cat. no. DY999; R\&D Systems) was added to the plate for 20 minutes at room temperature with slow rotation on a plate shaker. The reaction was stopped by the addition of $50 \mu \mathrm{l} 2 \mathrm{~N} \mathrm{H}_{2} \mathrm{SO}_{4}$ and the plate read at $450 \mathrm{~nm}$ on the SpectraMax Plus 384 plate reader (Molecular Devices, Sunnyvale, CA). PCSK9 values were calculated using the standard curve.

LDL Uptake Assay. A modified LDL uptake assay was carried out as previously described (Han et al., 2014). HepG2 cells overexpressing human PCSK9 were plated in a poly-D-lysine-coated 96-well black plate (BioCoat, cat. no. 354640; Corning, Teterboro, $\mathrm{NJ})$ and incubated with LY2584702 or 4-APP $(N=6)$ for 20 hours at $37^{\circ} \mathrm{C}$ and $5 \% \mathrm{CO}_{2}$ in a humidified chamber. Fluorescent LDL-BODIPY (cat. no. L-3483; Thermo Fisher Scientific) was then added to the assay and incubated with cells for an additional 4 hours at $37^{\circ} \mathrm{C}$ and $5 \% \mathrm{CO}_{2}$ in a humidified chamber. Cells were fixed with Prefer (cat. no. 414; Anatech Ltd., Battle Creek, MI) for 20 minutes at room temperature. Cells were washed twice with Dulbecco's phosphate-buffered saline without calcium chloride and magnesium chloride (DPBS; cat. no. 14190; Thermo Fisher Scientific) and were then treated with $0.01 \%$ Triton-X 100 (cat. no. T-9284; Sigma-Aldrich) diluted in DPBS for 15 minutes at room temperature. Cell nuclei were stained with $0.01 \mathrm{mg} / \mathrm{ml}$ propidium iodide (cat. no. P-3566; Thermo Fisher Scientific) diluted in DPBS. LDL uptake was determined using Acumen (TTP Labtech, Melbourn, UK) and presented as total fluorescence intensity verses total cell areas.

In Vivo Studies. All animal procedures were approved by and conducted in accordance with the Lilly and Covance Institutional Animal Care and Use Committee guidelines.

Rat Metabolism and Disposition Study with [ ${ }^{14}$ C]LY2584702. Male Sprague-Dawley rats were obtained from Hilltop Laboratory Animals, Inc. (Scottdale, PA). $\left[{ }^{14} \mathrm{C}\right]$ LY2584702 tosylate was administered by oral gavage to rats at a target dose of $50 \mathrm{mg} / \mathrm{kg}(1.14 \mu \mathrm{Ci} / \mathrm{mg})$ as a clear solution in 5\% vitamin $\mathrm{E}$ D- $\alpha$-tocopherol polyethylene glycol 1000 succinate, $1 \%$ hydroxyethyl-cellulose, and $0.05 \%$ Dow Corning Antifoam (cat. no. 1510-US, Midland, MI) in purified water. Three animals were sacrificed by exsanguination (cardiac puncture) under isoflurane anesthesia at 2 hours postdose. Blood (approximately 5-10 ml) was collected into tubes containing $\mathrm{K}_{2}$ EDTA anticoagulant from all animals at sacrifice and the resulting plasma was designated for metabolism profiling by HPLC radioprofiling and liquid chromatography-tandem mass spectrometry (Finnigan LCQ Advantage and Orbitrap instruments; Thermo Fisher Scientific).

In a separate group of intact animals, urine was collected in plastic containers surrounded by dry ice at $0-12$ hours and $12-24$ hours postdose, and at 24-hour intervals through 168 hours postdose. Feces were collected in plastic containers surrounded by dry ice at 24-hour intervals through 168 hours postdose. In bile duct-cannulated animals, urine was collected in plastic containers surrounded by dry ice at 0-12 and 12-24 hours postdose, and at 24-hour intervals through 72 hours postdose. Feces samples were collected in plastic containers surrounded by dry ice at 24 -hour intervals through 72 hours postdose. Bile was collected in plastic containers surrounded by dry ice at $0-6$, 6-12, and 12-24 hours postdose, and at 24-hour intervals through 72 hours postdose. The weights of all collected samples were recorded. Plasma, urine, feces, and bile samples were stored frozen at approximately $-70^{\circ} \mathrm{C}$ until analysis.
Lipid Metabolism Study in Long Evans Diet-Induced Obese Rats. Male Long Evans rats (Harlan Laboratories, Indianapolis, IN) were given food and water ad lib throughout the experiment unless otherwise indicated and kept on a 12-hour light/dark cycle. Animals were fed a high-energy diet (Teklad TD95217; Harlan Laboratories) for 10 weeks starting at age $4-5$ weeks to develop diet-induced obesity (DIO) and dyslipidemia. The compounds were then given for 4 weeks as the animals continued on high-fat diet feeding. Each compound $(N \sim 7-8)$ was administered to these diet-induced obese rats in a formulation of $1 \%$ hydroxyethylcellulose, $0.25 \%$ polysorbate 80 , and $0.05 \%$ Antifoam in purified water via oral gavage once daily at the indicated doses.

After 3 weeks of compound administration, we performed an oral olive oil challenge. Animals were fasted overnight and compound was administered at the indicated doses 4 hours prior to baseline blood collection. Olive oil bolus $(10 \mathrm{ml} / \mathrm{kg})$ was administered following the baseline blood collection. Blood samples were taken at 2, 4, and 6 hours after olive oil bolus. Food was returned following the final blood collection.

A tyloxapol infusion study was performed after 4 weeks of compound administration. Compound was administered at the indicated doses 4 hours prior to baseline blood collection and tyloxapol treatment. Food was removed at this time and the animals were fasted for the rest of the study. Four hours after compound dosing, baseline blood was collected and tyloxapol $(400 \mathrm{mg} / \mathrm{kg}$ at $5 \mathrm{ml} / \mathrm{kg}$, prepared in sterile saline) was given by slow intravenous infusion. Blood samples were taken at 1 and 2 hours after tyloxapol treatment. Animals were sacrificed after the last blood collection.

Lipoprotein Fractionation by HPLC. Lipoprotein fractions were analyzed by HPLC as previously described (Kieft et al., 1991). Briefly, $20-50 \mu \mathrm{l}$ of serum sample was applied to a Superose 10/300 GL size exclusion column (GE Healthcare Lifesciences, Port Washington, $\mathrm{NY}$ ) and eluted with phosphate-buffered saline, $\mathrm{pH} 7.4$ (diluted 1:10, cat. no. SH30258.01; HyClone, Logan, UT), containing 5 mM EDTA at $0.5 \mathrm{ml} / \mathrm{min}$. Cholesterol reagent (Cholesterol/HP Reagent 450061; Roche Diagnostics) at $0.12 \mathrm{ml} / \mathrm{min}$ was mixed with the column effluent through a T-connection, and the mixture was passed through a $15-\mathrm{m} \times$ $0.5-\mathrm{mm}$ internal diameter knitted tubing reactor (Aura Industries, New York, NY) immersed in a $37^{\circ} \mathrm{C}$ water bath. The colored product produced in the presence of cholesterol was monitored in the flow stream at $505 \mathrm{~nm}$. Analysis was done using ChemStation for LC Systems.

Liver Triglyceride Measurement. Liver triglycerides were measured using a commercial kit from Wako Diagnostics (cat. no. 46109092, Mountain View, CA) per the manufacturer's instruction. Briefly, about $0.05 \mathrm{~g}$ of liver was combined with $2 \mathrm{ml}$ of water in poly-Dlysine tube (cat. no. 6913-100; EMD Millipore, Temecula, CA), and homogenate was prepared using a Fast Prep homogenizer (FP120; Thermo Savant, Vernon Hills, IL). Standard was prepared by serial dilution of lipid calibrator (cat. no. 464-01601; Wako Diagnostics) in water. Standard and samples $(20 \mu \mathrm{l})$ were pipetted to a microplate, and $120 \mu \mathrm{l}$ of reagent $\mathrm{A}$ (preheated to $37^{\circ} \mathrm{C}$ ) was added to each well. After incubating for 10 minutes at $37^{\circ} \mathrm{C}, 80 \mu \mathrm{l}$ of reagent $\mathrm{B}$ was added to each well. Samples were well mixed and incubated at $37^{\circ} \mathrm{C}$ for 10 minutes. The absorbance was read at $600 / 700 \mathrm{~nm}$ using a SpectraMax M2 plate reader (Molecular Devices). The concentrations of liver triglycerides were calculated from the standard plot using appropriate dilution factor.

Lipidomics Analysis. Analysis of liver lipids was conducted via flow injection electrospray ionization-tandem mass spectrometry using an AB-SciEx 5600 TripleTOF as previously described (Newsom et al., 2016). The mass spectra were acquired in two stages. In the first stage the time-of-flight spectra were scanned with no fragmentation from 100 to 1200 daltons with a resolution of 40,000 . The second stage consisted of time-of-flight product ion scans of 611 precursor masses from 349.199-959.809 isolated with a resolution of 0.7 daltons and changed stepwise in 1.001-dalton increments. Sphingomyelins were identified and distinguished from phosphatidylcholine analytes by 
unfragmented proton adducts during the first stage. The remaining lipids were identified in the second stage by precursor and product ion pairs predicted by the analyte species and lipid class.

Liver tissue was homogenized to $50 \mathrm{mg} / \mathrm{ml}$ in isopropanol, then extracted along with an internal standard solution (143 $\mathrm{ng}$ of $\mathrm{D}_{5}$-triacyl glycerol 16:0, 16:0, 16:0; $509 \mathrm{ng}$ of phosphatidylcholine 14: $0,14: 0 ; 71.7 \mathrm{ng}$ sphingomyelin $17: 0 ; 7.50 \mathrm{ng}$ of ${ }^{15} \mathrm{~N},{ }^{13} \mathrm{C}_{3}$-Cer 16:0; $72.2 \mathrm{ng}$ of lysophosphatidylcholine 15:0; $27.1 \mathrm{ng}$ phosphotidylethanolamine 15:0, 15:0; and $15.0 \mathrm{ng}$ diacyl glyceride 15:0, 15:0 per mg of tissue) by a modified Bligh-Dyer extraction. The chloroform extract was combined with a spray solvent ( $20 \mathrm{mM}$ ammonium acetate in 1:1 isopropanol/ methanol) and injected directly into the instrument. Analyte concentrations were reported as an area ratio calculated as the analyte peak area normalized to the internal standard peak area.

Gene Expression Analysis of Liver Samples. Liver tissues from LE-DIO rats were clamp frozen. Total RNA was isolated using QIAGEN RNeasy Mini Kit (cat. no. 74104). cDNA was synthesized using SuperScript III First-Strand Synthesis System for RT-PCR (cat. no. 18080051; Thermo Fisher Scientific). Real-time PCR was carried out using the 7900 HT Sequence Detection System from Applied Biosystems per manufacturer's instructions. All reagents were from Thermo Fisher Scientific. These include TaqMan Gene Expression Master Mix (cat. no. 4369016); primer and probe mixed for $18 \mathrm{~S}$ rRNA (cat. no. 4310893E); rat ACC1 (Rn00573474_m1); rat ACLY (Rn00566411_m1); rat FASN mRNA (Rn01463550_m1); rat SCD1 mRNA (Rn00594894_g1); rat PCSK9 mRNA (Rn01416753_m1); rat HMGCR (Rn00565598_m1); rat low-density lipoprotein receptor mRNA (LDLR, Rn00598442-m1), rat ATP binding cassette subfamily G member 5 (ABCG5, Rn01499073_m1), rat ATP binding cassette subfamily G member 8 (ABCG8, Rn00590367_m1), cytochrome P450 family 7 subfamily A member 1 (Cyp7a1, Rn00564065_m1).

Clinical Pathology Methods. Serum levels of triglycerides (cat. no. 11877771), total bilirubin (cat. no. 11822713), alkaline phosphatase (cat. no. 12172933), alanine aminotransferase (ALT) (cat. no. 11876805), aspartate aminotransferase (cat. no. 11876848), albumin (cat. no. 11970909), and total protein (cat. no. 11929917) were all determined using the Roche Hitachi Modular P System Analyzer (Roche Diagnostics). All kits were purchased from Roche and run per manufacturer's instructions.

\section{Results}

LY2584702 Significantly Lowered LDL-C in Long Evans Diet-Induced Obese Rat Model. To reversetranslate the clinical efficacy of LY2584702 to a preclinical model, we selected the Long Evans diet-induced obese (LE-DIO) rat model for the study. LE-DIO rats develop significant obesity and dyslipidemia when fed a high-energy $\operatorname{diet}(\mathrm{Li}$ et al., 2008). LY2584702 was administered orally to the animals at 15 or $50 \mathrm{mg} / \mathrm{kg}$ (mpk) per day for 4 weeks. LY2584702 was well tolerated at both doses with no clinical signs of distress. Slight reductions in average daily food intake were observed $(-10.9 \%$ and $-14.1 \%$ for $15 \mathrm{mpk}$ and $50 \mathrm{mpk}$ of LY2584702 treatment groups, respectively), but this did not result in body weight reductions (data not shown). Nonfasting serum samples were collected at 4 hours after compound administration on study day 29. Lipoprotein changes were analyzed by HPLC. As shown in Fig. 1, A and B, significant reductions in VLDL-C $(-43.2 \%,-64.2 \%)$ and LDL-C $(-45.3 \%,-60.2 \%)$ were observed with $15 \mathrm{mpk}$ and $50 \mathrm{mpk}$ of LY2584702. Reduction in HDL-C (-20.6\%, -39.9\%) was also observed for the two compound-treated groups, although to a lesser extent compared with reductions in LDL-C (Fig. 1C). Interestingly serum triglycerides (TG) were not changed by administration of either dose level of LY2584702 (Fig. 1D). Serum PCSK9 levels were modestly reduced $(-16.0 \%$ and $-17.6 \%$ for $15 \mathrm{mpk}$ and $50 \mathrm{mpk}$ of LY2584702, respectively; Fig. 1E). The hypolipidemic effects of LY2584702 also appeared to be time-dependent. In a separate study, we treated LE-DIO rats with $25 \mathrm{mpk}$ and $50 \mathrm{mpk}$ of LY2584702 for 1 week. Only slight reductions in LDL-C were observed (-10.6\% and $-25.5 \%$ for $25 \mathrm{mpk}$ and $50 \mathrm{mpk}$ of LY2584702, respectively; Supplemental Table 1). Similarly, lesser changes in VLDL-C, HDL-C, serum PCSK9, and liver TG were observed after 1 week compared with 4 weeks of treatment (Supplemental Table 1 and Fig. 1). Together, these data suggest treatment with LY2584702 in the LE-DIO rat model resulted in time- and dosedependent lowering of lipids, particularly LDL-C.

Surprisingly, we also observed dose-dependent increase in liver TG (1.9- and 2.8-fold versus vehicle for $15-\mathrm{mpk}$ and 50-mpk-LY2584702 groups, respectively; Fig. 1F) after 4 weeks of compound administration. This increase in liver TG is inconsistent with a literature report on p70S6K1 knockdown in mice (Li et al., 2011). We hypothesized this increase in liver TG may be attributed to off-target activity of LY2584702 or the result of a metabolite.

Metabolite Identification of 4-APP after Oral Administration of LY2584702 in Rats. The pharmacokinetics, distribution, excretion, and metabolism of LY2584702 were evaluated in Sprague-Dawley intact and bile duct-cannulated rats following a single oral dose of $50 \mathrm{mg} / \mathrm{kg}$ of $\left[{ }^{14} \mathrm{C}\right] \mathrm{LY} 2584702$
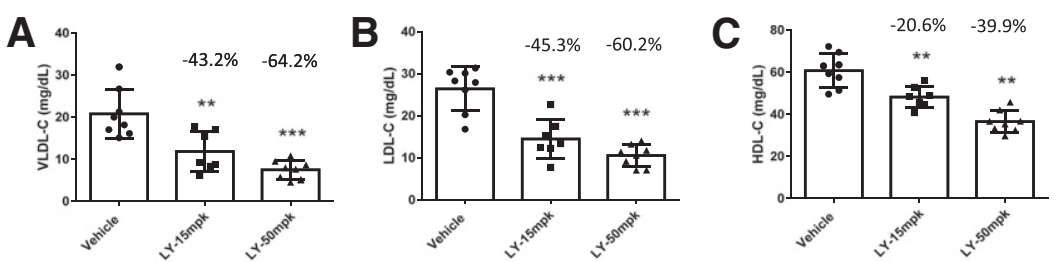

D

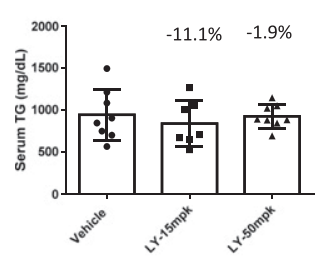

$E$

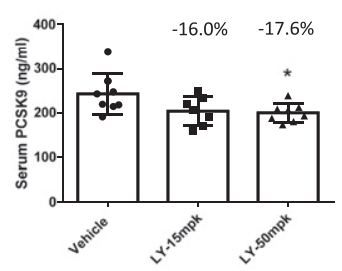

$\mathbf{F}$

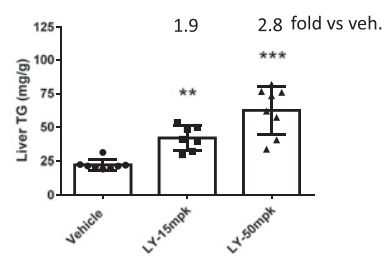

Fig. 1. Effects of LY2584702 (LY) on lipid related endpoints in LE-DIO rat model. Vehicle, $15 \mathrm{mpk}$ of $\mathrm{LY}$, and $50 \mathrm{mpk}$ of LY were administered to LE-DIO rats ( $N=8$ per treatment group) daily via oral gavage for 4 weeks. Lipid related endpoints were measured at 4 hours after last dose without fasting. (A) Serum VLDL-C. (B) Serum LDL-C. (C) Serum HDL-C. (D) Serum triglycerides. (E) Serum PCSK9. (F) Liver triglyceride content. Error bars depict standard deviation. $P$ values were calculated using one way ANOVA followed by Dunnett's test. ${ }^{*} P<0.05$ versus vehicle; $* * P<0.01$ versus vehicle; $* * * P<0.001$ versus vehicle. 
administered as the tosylate salt. The chemical structures of $\left[{ }^{14} \mathrm{C}\right] \mathrm{LY} 2584702$ and a potential metabolite 4-aminopyrazolo[3,4-d]pyrimidine (4-APP) are shown in Fig. 2A. The radiolabel was inserted at the $\mathrm{C} 2$ carbon in the pyrimidine ring. In plasma, the parent drug was the largest radiocomponent observed for all time points examined $(0.5,1-, 2-, 4-, 8-$, and 12-hour samples), representing $94,96,90,87,82$, and $72 \%$ of the circulating radioactivity, respectively. Four metabolites of LY2584702 were identified in plasma, including 4-APP (A18 in Fig. 2), which resulted from apparent N,N-dealkylation of the piperidine ring nitrogen. 4-APP was first detected at the 1-hour time point and represented $3 \%$ of the circulating radioactivity up to 12 hours (Fig. 2B). Radioprofiling of feces from the bile duct-intact rats (0-48 hours) revealed the presence of parent drug and 11 metabolites, with 4-APP (A18) representing 4\% of the dose within the first 24 hours of fecal collection (Fig. 2C). Radioprofiling of bile from the bile duct-cannulated rats revealed the presence of parent drug and 16 metabolites, but 4-APP itself was not detected in bile. This observation suggests that either LY2584702 may degrade to 4-APP in feces, or biliary secretion of 4-APP from plasma is followed by rapid elimination into feces. Alternatively, 4-APP may be subsequently metabolized and rapidly eliminated. Radioprofiling of urine from bile duct-intact rats revealed the presence of 12 components, but 4-APP was not detected in that matrix. Thus, results from the oral disposition study with $\left[{ }^{14} \mathrm{C}\right] \mathrm{LY} 2584702$ in rats confirm that 4 -APP is generated as a circulating metabolite in that species.

4-APP is an adenine nucleic acid analog originally investigated for the treatment of cancer (Shaw et al., 1960). Preclinical studies with 4-APP revealed hepatic fat accumulation in multiple species (Shaw et al., 1960). Interestingly, it was also reported that when administered intraperitoneally at 5-50 mpk, 4-APP acutely and markedly lowered serum triglyceride and total cholesterol levels (Shiff et al., 1971). These data prompted us to ask whether 4-APP can be formed in the LE-DIO rats after administration of LY2584702 and may to some degree contribute to the lipid-lowering efficacy.

Formation of 4-APP from LY2584702 in LE-DIO Rats. A bioanalytical method was specifically developed to quantify concentrations of 4-APP in serum and liver samples after treatment with $\sim 15-50 \mathrm{mpk}$ of LY2584702 for 1 week or 4 weeks. LY2584702 was detected in both serum (Fig. 3A, open bars) and liver (Fig. 3A, filled bars) at both time points. Liver concentrations of LY2584702 were higher than concentrations in serum. As shown in Fig. 3B, 4-APP was detected in both serum (open bars) and liver (filled bars) after 1 week of
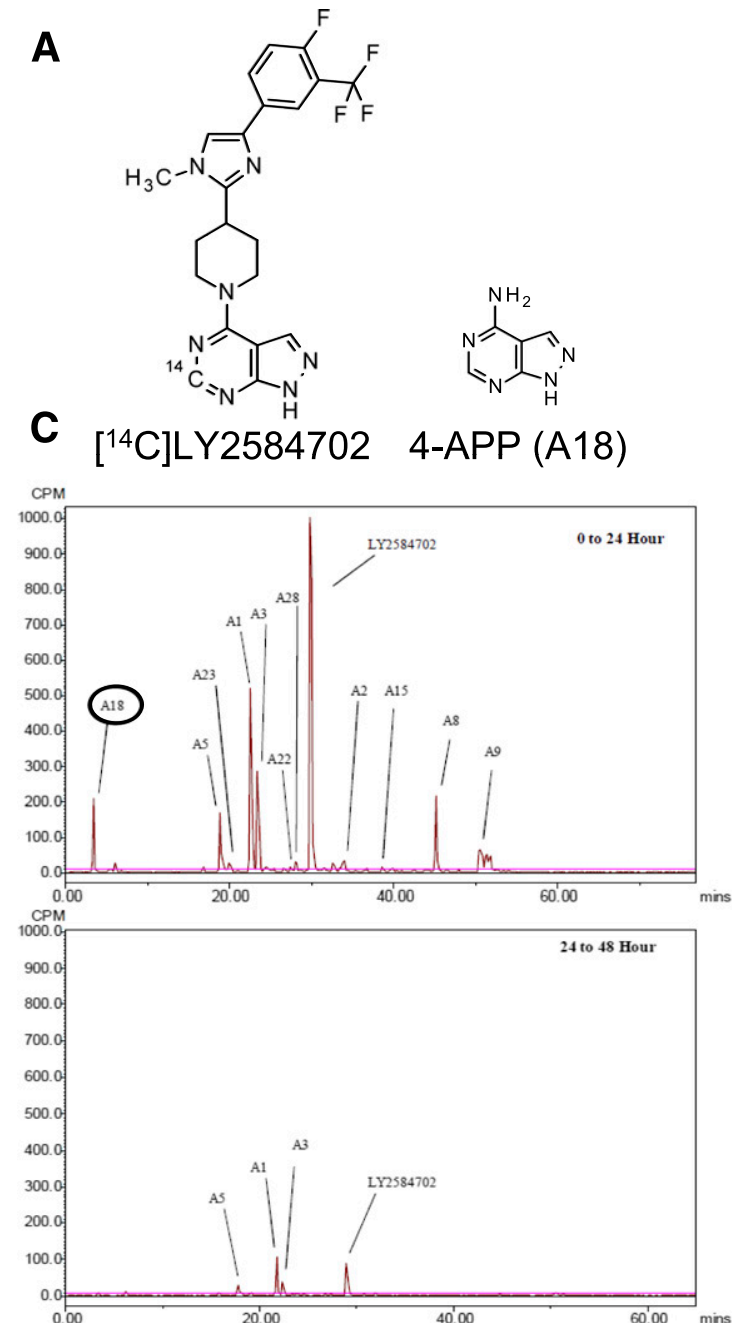

B
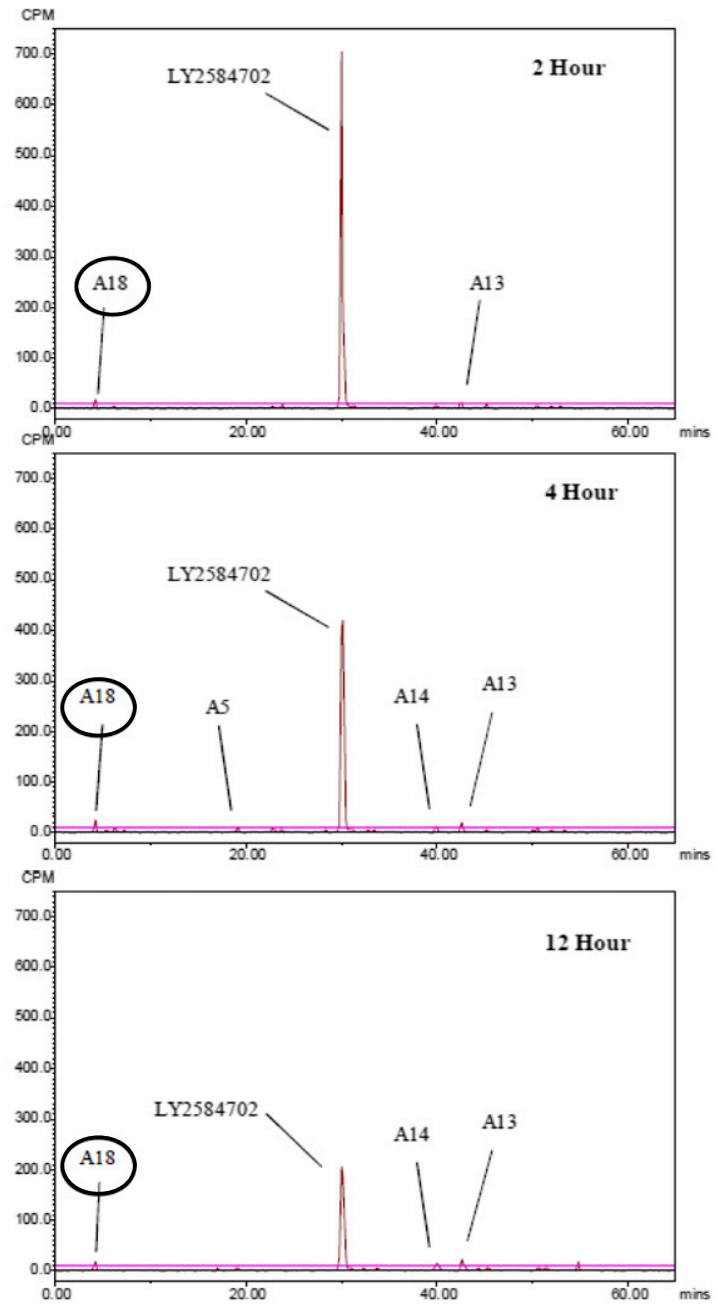

Fig. 2. Reconstructed radioprofiles from intact Sprague-Dawley rats after a single oral dose of $50 \mathrm{mpk}$ of $\left[{ }^{14} \mathrm{C}\right] \mathrm{LY} 2584702$. (A) Chemical structures of LY2584702 and 4-APP. (B) Pooled 2-, 4-, and 12-hour plasma samples. (C) Pooled 0-24 hour and $24-48$ hour fecal samples. (Note: A18 = 4-APP.) 
administration of LY2584702. As with LY2584702, liver concentrations of 4-APP were higher than those in the serum. Interestingly, liver 4-APP concentrations continued to increase after 4 weeks of LY2584702 administration, whereas serum concentrations remained low (Fig. 3B). These data suggest that 4-APP was formed as a metabolic product of LY2584702 in the liver. The relative molar quantity of hepatic levels of 4-APP versus LY2584702 increased from $0.5 \%$ at week 1 to $5.2 \%$ at week 4 in the 50-mpk-LY2584702 treatment groups. Total concentrations of 4-APP were higher in the liver than in serum and increased over time, reaching $1160 \mathrm{nM}$ after 4 weeks of treatment with $50 \mathrm{mpk}$ of LY2584702. Correcting for unbound fraction of 4-APP in both matrices, the liver/serum ratio of 4-APP at 4 hours postdose on day 29 in this treatment group was $12.7 \pm 5.3$.

Comparisons between LY2584702, 4-APP, and MTP Inhibitor Lomitapibe in LE-DIO Rats. It was previously reported that when administered intraperitoneally at 5-50 mpk, 4-APP acutely and markedly suppressed hepatic VLDL secretion and intestinal chylomicron production (Krause et al., 1981; Shiff et al., 1971). This activity profile of high-dose 4-APP is distinct from the observation with LY2584702 in LE-DIO rats that nonfasting serum triglyceride levels were minimally affected (Fig. 1D). To understand how low-dose 4-APP may impact lipid metabolism when dosed orally, 4-APP was tested at $0.5 \mathrm{mpk}$ in the LE-DIO rat model in parallel with $50 \mathrm{mpk}$ of LY2584702. The 4-APP dose level of $0.5 \mathrm{mpk}$ was selected on the basis of hepatic safety concerns anticipated with long-term dosing (Shaw et al., 1960; Henderson, 1963). The material safety data sheet indicates that 4-APP has an oral $\mathrm{LD}_{50}$ of $141 \mathrm{mg} / \mathrm{kg}$ in the rat.

For comparison, an MTP inhibitor lomitapibe (MTPi) was also included in the study at 1-mpk dose level (Funatsu et al., 2002). After 3 weeks of once-daily dosing, animals were fasted overnight and serum samples were collected and subjected to lipoprotein analysis by HPLC. As shown in Fig. 4A, 0.5-mpk4-APP treatment group demonstrated remarkably similar lipoprotein profiles compared with the 50-mpk-LY2584702 treatment group. These lipoprotein profiles differ from those observed in the MTPi-treated group. Specifically, MTPi most significantly suppressed VLDL-C by $90.9 \%$, whereas LY2584702 and 4-APP modestly suppressed VLDL-C by $46.8 \%$ and $31.0 \%$, respectively (Fig. 4B). All three compounds lowered LDL-C to a similar extent, reaching 70.0\%, 58.4\%, and $58.0 \%$ for the MTPi, LY2584702, and 4-APP groups, respectively (Fig. 4C). Reduction in HDL-C was modest for all three treatment groups (Fig. 4D). Interestingly, the HDL fraction in MTPi-treated group shifted slightly left on the chromatogram compared with the vehicle group (Fig. 4A), indicating potential increase in HDL particle sizes. This phenomenon was not observed with LY2584702 or 4-APP treatment groups. Serum PCSK9 levels were not affected by MTPi treatment, whereas the 4-APP treatment group showed a very modest reduction in PCSK9 compared with vehicle (-24.3\%, Fig. $4 \mathrm{E})$.

All three compounds were well tolerated in the 4-week study. No significant changes in food intake or body weight were observed for the duration of the study (data not shown). Other than a slight increase in serum ALT levels in the MTPitreated group, there were no significant changes in a panel of hepatobiliary biomarkers including ALT, aspartate aminotransferase, alkaline phosphatase, total protein, and albumin after 3 weeks of treatment (Supplemental Table 2).

Minimal Effects of LY2584702 and Low-Dose 4-APP on Chylomicron-TG and VLDL-TG Secretion. We conducted kinetic studies to probe how LY2584702 and low-dose 4-APP regulate lipoprotein metabolism. An oil fat tolerance test was performed on animals fasted overnight following 3 weeks of oral administration of $1 \mathrm{mpk}$ of MTPi, $50 \mathrm{mpk}$ of LY2584702, and $0.5 \mathrm{mpk}$ of 4-APP. Consistent with the changes in VLDL-C (Fig. 4B), baseline (time 0) serum TG were significantly suppressed by MTPi (-91.8\%, Fig. 5A) but modestly suppressed by LY2584702 and 4-APP (-46.9\% and $-39.1 \%$, respectively, Fig. 5A). Following an oral olive oil bolus, serum TG excursions were monitored. No effects on serum TG excursion were observed in animals treated with LY2584702 or 4-APP compared with vehicle-treated animals (increased by $1.4 \%$ and $0.1 \%$ in area under the curve, respectively), whereas MTPi significantly suppressed serum TG excursion (-55.9\% in area under the curve) (Fig. 5, B and C). Studies on VLDL secretion were conducted after 4 weeks of oral administration of $1 \mathrm{mpk}$ of MTPi, $50 \mathrm{mpk}$ of LY2584702, and $0.5 \mathrm{mpk}$ of 4 -APP. Animals were fasted for 4 hours prior to tyloxapol infusion. Baseline (time 0) serum TG were significantly suppressed by MTPi (-92.8\%, Fig. 5D) but modestly suppressed by LY2584702 and 4-APP (-19.0\% and $-41.5 \%$, respectively, Fig. 5D). Following tyloxapol infusion, serum TG rapidly increased in the vehicle group (Fig. 5E). LY2584702 and 4-APP modestly inhibited serum TG increase $(-15.8 \%$ and $-26.8 \%$, respectively, Fig. 5, E and F), whereas MTPi significantly inhibited serum TG increase (-65.6\%, Fig. 5, E and F). Together, these data suggest that LY2584702 and low-dose 4-APP did not impact intestinal chylomicron-TG secretion and only modestly inhibited hepatic VLDL-TG secretion.

Hepatic Lipid and Gene Expression Changes in LE-DIO Rats after Treatment with LY2584702, 4-APP, or MTP Inhibitor Lomitapibe. To compare how LY2584702, 4-APP, and MTPi affect hepatic lipids after 4 weeks of oral administration, we first measured liver TG content by a
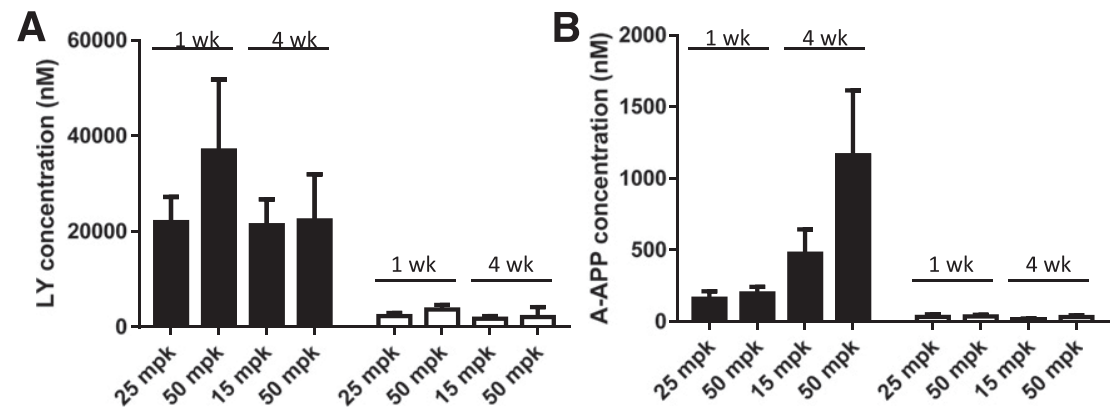

Fig. 3. Formation of 4-APP from LY2584702 (LY) in LE-DIO rats. Data were collected from two separate in vivo studies. In the first study, $25 \mathrm{mpk}$ of $\mathrm{LY}, 50 \mathrm{mpk}$ of LY were administered to LE-DIO rats $(N=7$ per treatment group) daily via oral gavage for 1 week. In the second study, $15 \mathrm{mpk}$ of LY, $50 \mathrm{mpk}$ of LY were administered to LE-DIO rats $(N=8$ per treatment group) daily via oral gavage for 4 weeks. Liver (filled bars) and serum (open bars) compound exposure for LY and 4-APP were monitored at 4 hours after last dose. (A) Liver and serum concentrations of LY. (B) Liver and serum concentrations of 4-APP. Error bars depict standard deviation. 


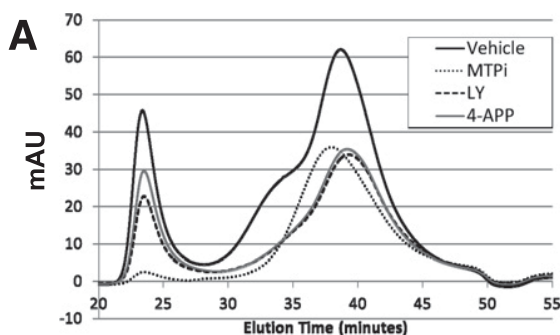

C

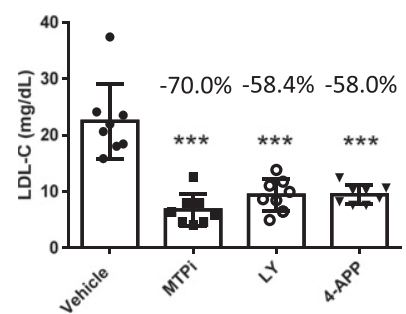

D

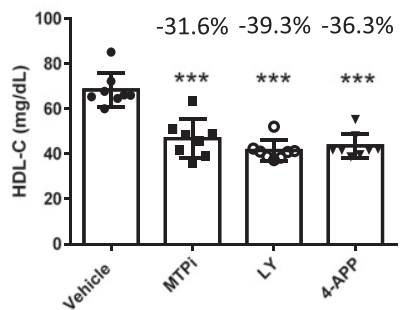

B

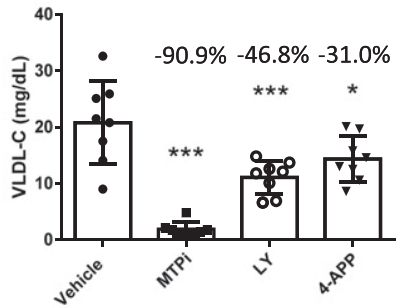

E

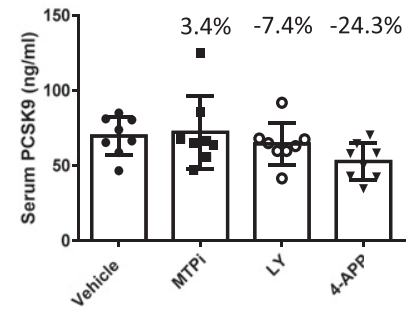

Fig. 4. Effects of LY2584702 (LY), 4-APP, and MTP inhibitor lomitapibe (MTPi) on lipoprotein profile in LE-DIO rat model. Vehicle, 1 mpk of MTPi, $50 \mathrm{mpk}$ of LY, or $0.5 \mathrm{mpk}$ of 4-APP was administered to LE-DIO rats $(N=8$ per treatment group) daily via oral gavage for 3 weeks. Serum samples were obtained at 4 hours postdose from animals fasted overnight and subject to lipoprotein analysis via HPLC. (A) HPLC chromatograms of cholesterol content of lipoprotein fractions. (B) Serum VLDL-C. (C) Serum LDL-C. (D) Serum HDL-C. (E) Serum PCSK9. Error bars depict standard deviation. $P$ values were calculated using one way ANOVA followed by Dunnett's test. ${ }^{*} P<0.05, * * P<0.01,{ }^{*} * * P<0.001$ versus vehicle.

biochemical method using the Wako Diagnostics kit. Consistent with their differential effects on VLDL secretion, the 1-mpk-MTPi treatment group showed a dramatic increase in liver TG levels after 4 weeks of compound administration (11.9fold versus vehicle), whereas $50 \mathrm{mpk}$ of LY2584702 and $0.5 \mathrm{mpk}$ of 4-APP treatment groups each demonstrated more modest increase in liver TG levels (4.1- and 3.4-fold versus vehicle,
Fig. 6A). To further confirm these findings, we carried out a lipidomics analysis on the liver samples following extraction with organic solvent. Liver total TG content by this measurement closely aligns with the data derived from biochemical measurement (data not shown). Semiquantitative data of 10 types of lipids are shown in Fig. 6B. Both total TG and total cholesterol ester (CE) were significantly increased in the MTPi
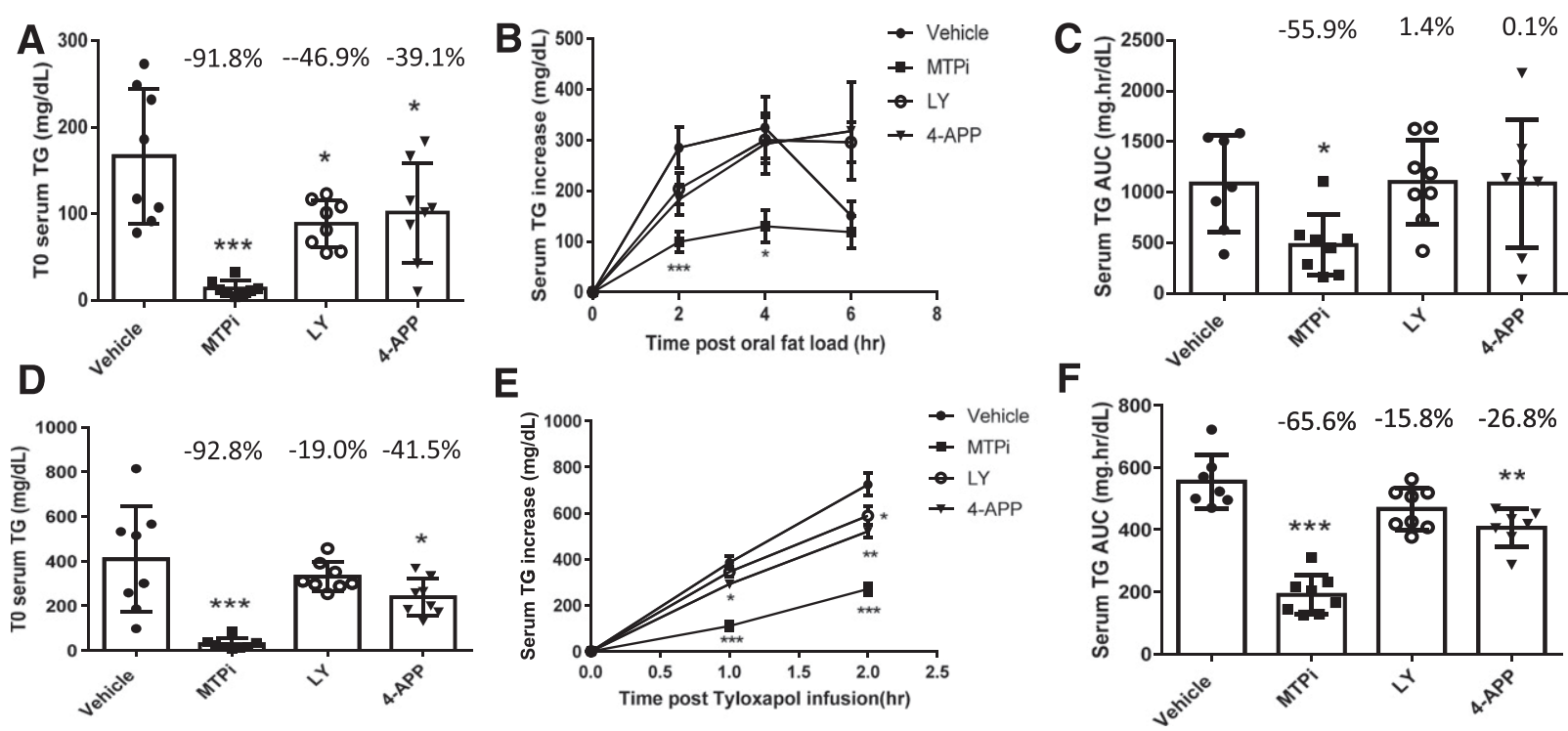

Fig. 5. Effects of LY2584702 (LY), 4-APP, and MTP inhibitor lomitapibe (MTPi) on chylomicron-TG secretion and VLDL-TG secretion in LE-DIO rat model. Vehicle, $1 \mathrm{mpk}$ of MTPi, $50 \mathrm{mpk}$ of LY, or $0.5 \mathrm{mpk}$ of 4 -APP was administered to LE-DIO rats $(N=8$ per treatment group) daily via oral gavage for 4 weeks. On day 21, animals were challenged with oral olive oil bolus following overnight fasting. (A) Serum TG after overnight fasting immediately prior to olive oil bolus (To: time zero). (B) Serum TG increase (from time point 0 ) at 2, 4, and 6 hours after olive oil bolus. (C) Area under the curve for serum TG (AUC) following oral fat challenge. In (A-C), one animal in the vehicle group was excluded from the analysis owing to technical failure in olive oil gavage. (D-F) On day 28, animals were fasted for 4 hours and infused with $400 \mathrm{mpk}$ of tyloxapol to inhibit lipolysis of TG via lipoprotein lipase. (D) Serum TG after 4 hours of fasting immediately prior to tyloxapol infusion. (E) Serum TG increase (from 0 time point) at 1 and 2 hours after tyloxapol infusion. (F) Area under the curve for serum TG (AUC) following tyloxapol infusion. In (E) and (F), one animal in the 4-APP treatment group was excluded from the analysis owing to technical failure in tyloxapol infusion. 
treatment group, consistent with MTPi's mode of action. LY2584702 and 4-APP treatment groups showed modest increase in both TG and CE, also consistent with their modest effects on VLDL secretion. Most of the other types of lipids were only minimally affected, with the exception of total PE and total lysophosphotidylethanolamine (LPE). Interestingly, both LY2584702 and 4-APP treatment increased PE and LPE by about 2-fold, whereas the MTPi treatment had minimal effect. This data further supports the notion that LY2584702 and 4-APP share similar pharmacology profiles distinct from that of MTPi.

To understand the potential mechanism related to these lipid changes, we next evaluated mRNA expression levels of a panel of genes involved in lipid metabolism. As shown in Fig. 6C, treatment with MTPi, LY2584702, or 4-APP all significantly suppressed mRNA levels of a list of de novo lipogenic genes, including ACC1, ACLY, FASN, and SCD1. The 4-APP treatment group also showed significant suppression of several SREBP2 genes, including HMGCR, LDLR, and PCSK9.

LY2584702 treatment group showed a trend in suppressing HMGCR and PCSK9. We also evaluated several genes involved in cholesterol/bile acid metabolism, including ATP binding cassette subfamily $G$ members 5 and 8 (ABCG5, ABCG8) and cytochrome P450 family 7 subfamily A member
1 (Cyp7a1). All three genes showed a trend of increase after treatment with MTPi, LY2584702, or 4-APP, with changes in ABCG5 and Cyp7a1 reaching statistical significance for the MTPi treatment group. These data suggests the possibility of increase in biliary and fecal cholesterol/bile acid excretion following compound treatment.

The MTPi treatment group had significantly greater accumulation of neutral lipids in the liver (Fig. 6, A and B), which could lead to feedback regulation of gene expression. The mechanism for how LY2584702 regulates gene expression is not entirely clear. On the basis of the LY2584702 concentrations in the liver, P70S6K1 is predicted to be only partially inhibited throughout the course of the study, with $\sim 50 \%$ inhibition at the time point when the pharmacology endpoints were taken (Supplemental Fig. 1). Inhibition of P70S6K1 may partly contribute to the suppression of de novo lipogenic genes via inhibition of SREBP1c (Li et al., 2011). However, 4-APP did not inhibit P70S6K1 (Supplemental Fig. 1A). The mechanism for how 4-APP treatment regulates expression of lipogenic genes remains to be determined. The similar patterns of regulation in hepatic lipids and gene expression following LY2584702 and 4-APP treatment further support the notion that 4-APP, as a metabolite of LY2584702 in the liver, may contribute to its observed pharmacology.
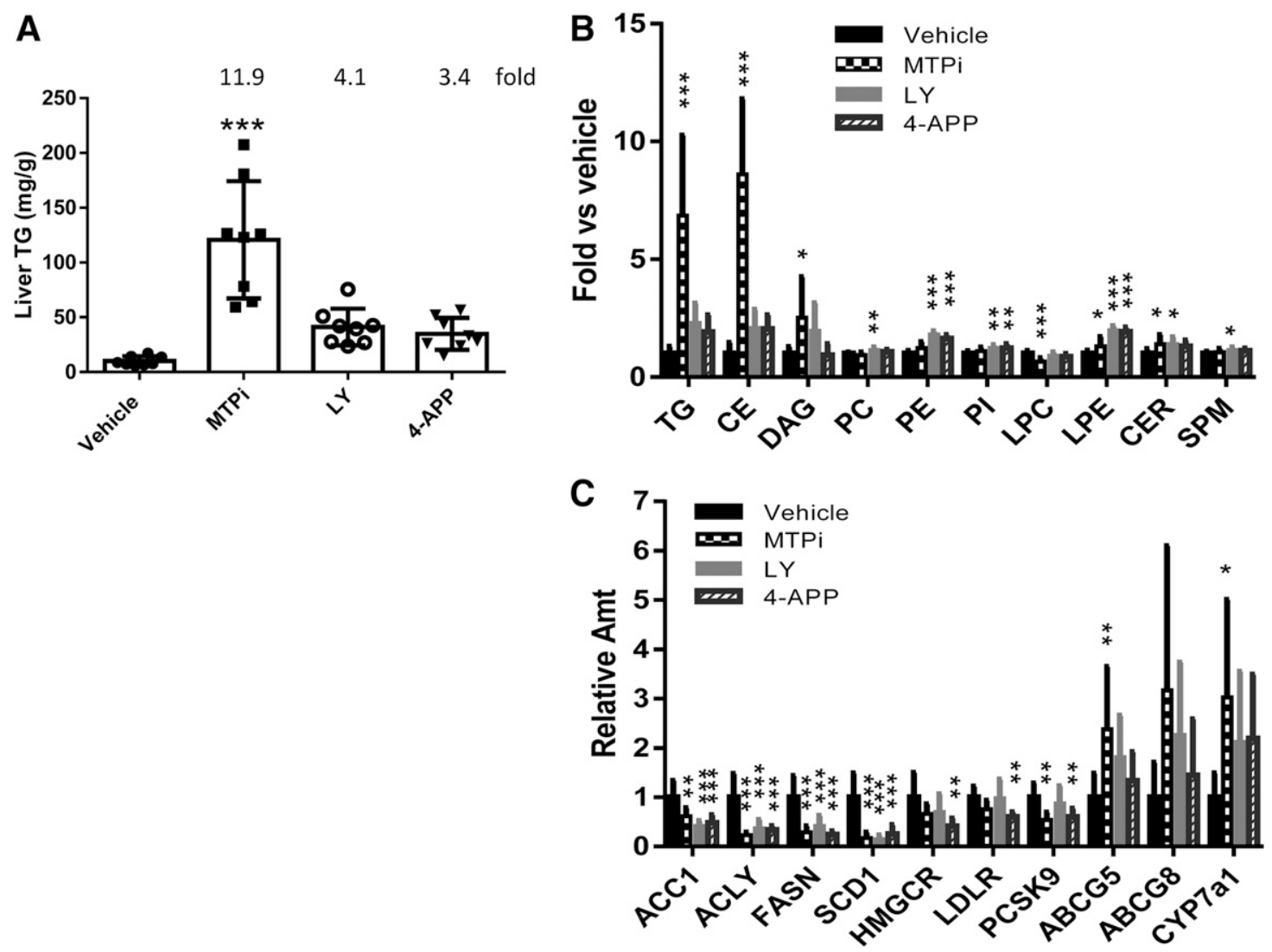

Fig. 6. Effects of LY2584702 (LY), 4-APP, and MTP inhibitor lomitapibe (MTPi) on hepatic lipids and mRNA expression in LE-DIO rat model. Vehicle, $1 \mathrm{mpk}$ of MTPi, $50 \mathrm{mpk}$ of LY, or $0.5 \mathrm{mpk}$ of 4 -APP was administered to LE-DIO rats $(N=8$ per treatment group) daily via oral gavage for 4 weeks. Four hours postdose on day 29, all animals were sacrificed and liver samples were analyzed for lipid content and gene expression. (A) Liver TG levels measured by biochemical method using the Wako Diagnostics kit. (B) Relative levels of 10 types of lipids in liver measured by lipidomics method. (C) Hepatic mRNA relative expression of lipid metabolism genes. Error bars depict standard deviation. $P$ values were calculated using one-way analysis of variance followed by Dunnett's test. ${ }^{*} P<0.05, * * P<0.01, * * * P<0.001$ versus vehicle. DAG, diacyl glyceride; LY, PC, phosphatidylcholine; PI, phosphatidylinositol; LPC, lysophosphatidylcholine; CER, ceramide; SPM, sphingomyelin. 
4-APP versus LY2584702 Treatment on LDL Uptake and Gene Expression in HepG2 Cells. Using human hepatoma HepG2 cells overexpressing high levels of exogenous PCSK9, we next asked whether LY2584702 and low-dose 4-APP may enhance LDL clearance. Compared with normal HepG2 cells, these PCSK9 overexpressing cells have lower baseline levels of LDL uptake owing to suppressed LDLR protein, resulting in higher assay sensitivity and reproducibility in our hands. As shown in Fig. 7A, 24-hour treatment with low concentrations of 4-APP $(0.08-2 \mu \mathrm{M})$ dose dependently enhanced uptake of Bodipy-labeled LDL particles into the HepG2 cells. In contrast, treatment with $10 \mu \mathrm{M}$ LY2584702 slightly reduced LDL uptake in this system (Fig. 7A). These observations were confirmed in several independent studies. These data suggest 4-APP may be the active entity that may enhance LDL clearance, leading to lowering of LDL in vivo in both rat model and humans.

We next tested these two compounds for their effects on mRNA expression in HepG2 cells (Fig. 7B). Treatment of HepG2 cells with 4-APP for 24 hours led to significant reduction of mRNA levels of a panel of lipogenic genes, including ACC1, ACLY, FASN, and SCD1. 4-APP treatment also significantly reduced mRNA levels of PCSK9 and HMGCR (Fig. 7B). In the same study, treatment with $10 \mu \mathrm{M}$ LY2584702 showed a similar trend in reducing mRNA levels of PCSK9 and the lipogenic genes. However, the magnitude of mRNA suppression is generally less in the LY2584702-treated group compared with the 4-APP-treated groups. Interestingly, mRNA levels of LDLR were dose dependently increased with 4-APP treatment compared with dimethyl sulfoxide control (Fig. 7B), whereas LY2584702 treatment did not change LDLR mRNA levels (Fig. 7B). A similar observation was made in HepG2 cells with PCSK9 overexpression (Supplemental Fig. 2). The inhibition of lipogenic genes was generally less robust and induction of LDLR mRNA was more significant, probably as a result of experimental variation. Endogenous PCSK9 mRNA cannot be assessed in this system owing to interference from the overexpressed PCSK9.

\section{Discussion}

It has been reported previously that LY2584702, a selective inhibitor of p70S6K1, lowered serum total cholesterol, LDL cholesterol, and triglyceride in cancer patients (Hollebecque et al., 2014; Tolcher et al., 2014). This hypolipidemic effect of LY2584702 was recapitulated in a rodent model of metabolic syndrome: Long Evans diet-induced obese rats. Reduction in serum LDL-C was observed in a time- and dose-dependent manner after 4 weeks of treatment with LY2584702 (Fig. 1, Supplemental Table 1). Serum PCSK9 was reduced in LE-DIO rats after 4 weeks of treatment with LY2584702, but to a lesser extent compared with observed changes in LDL-C (Fig. 1E). Whether this small reduction in PCSK9 may have a direct and meaningful impact on LDL clearance is not clear.

Surprisingly, we also observed significant increases in liver TG in this model following LY2584702 treatment (Fig. 1F). These data contradict the genetic data from the knockdown of p70S6K1, the known biologic target of LY2584702, that led to reduction in liver TG in mice (Li et al., 2011). We hypothesized this increase in liver TG may be attributed to off-target activity of LY2584702 or the result of formation of a drug metabolite.

Metabolite identification in Sprague-Dawley rats confirmed that 4-APP is generated as a metabolite in the plasma and feces following a single dose of $\left[{ }^{14} \mathrm{C}\right] \mathrm{LY} 2584702$ (Fig. 2). In fact, 4 -APP circulated in plasma and comprised $4 \%$ of the dose in feces collected within the first 24 hours postdose. In LE-DIO rats administered LY2584702 for 1 week or 4 weeks, 4-APP was detected and quantified in both serum and liver. Total concentrations of 4-APP were significantly higher in the liver than in serum and increased after 4 weeks of treatment with LY2584702. Accumulation of 4-APP in the liver would probably impact hepatic lipid metabolism.

4-APP is a purine nucleic acid analog previously shown to have hypolipidemic effects in rodents. High doses of 4-APP, when administered intraperitoneally, acutely shut down hepatic VLDL secretion and intestinal chylomicron secretion (Krause et al., 1981; Shiff et al., 1971). However, these dose levels were associated with significant toxicity when administered for longer than 2 weeks (Shaw et al., 1960). It was intriguing, however, that oral administration of up to 0.15 mpk of 4-APP in cancer patients for 2 weeks was well tolerated and serum cholesterol was reported to remain normal in these patients (Shaw et al., 1960). Whether oral administration of low-dose 4-APP could produce similar hypolipidemic effects in rodents was not initially clear.
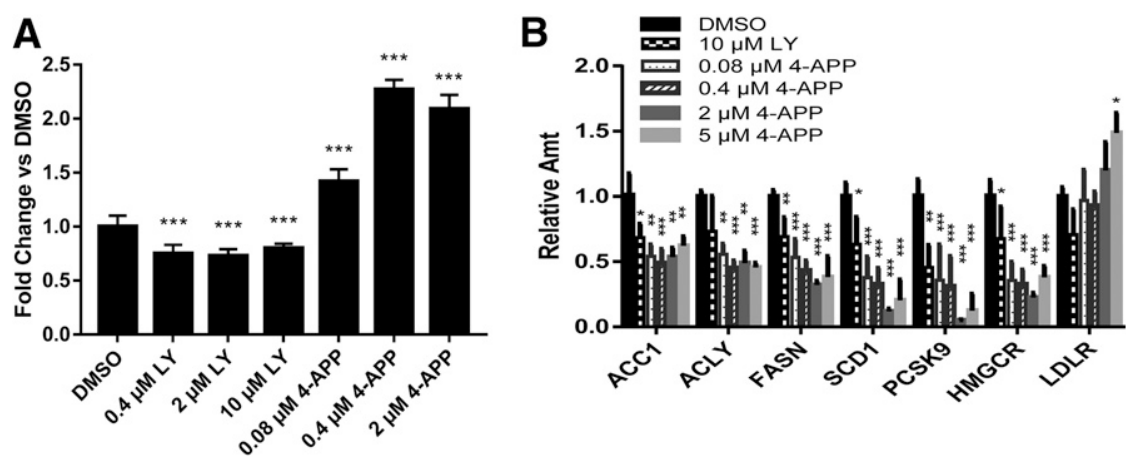

Fig. 7. Effects of LY2584702 (LY) and 4-APP on LDL uptake and gene expression in HepG2 cells. (A) HepG2 cells with overexpression of human PCSK9 were treated with LY and 4-APP at the indicated concentrations for 20 hours followed by incubation with Bodipy-labeled LDL for additional 4 hours. Efficiency of LDL uptake was assessed by fluorescence intensity normalized by cell areas and presented as fold changes over dimethyl sulfoxide (DMSO) control. (B) HepG2 cells were treated with LY2584702 and 4-APP for 24 hours at the indicated concentrations. mRNA levels of lipid metabolism genes were measured by quantitative PCR. Error bars depict standard deviation. $P$ values were calculated using one way ANOVA followed by Dunnett's test. $* P<0.05,{ }^{*} * P<0.01,{ }^{*} * * P<0.001$ versus DMSO control. 
In this preclinical investigation, we report that chronic oral administration of low-dose 4-APP effectively lowered LDL cholesterol with minimal impact on hepatobiliary biomarkers (Fig. 4, Supplemental Table 2). Lipoprotein profile changes were remarkably similar between treatments with 4-APP and LY2584702. Specifically, after 3 weeks of treatment, both LY2584702 and 4-APP produced very significant reduction in LDL-C (Fig. 4C). VLDL-C was modestly reduced (Fig. 4B). These lipoprotein profile changes are distinctly different from changes elicited by an MTP inhibitor, lomitapibe, in which the most significant inhibition is on the VLDL fraction.

As with the time-dependent pharmacology observed with LY2584702, low-dose 4-APP also lowered serum lipids in a time-dependent manner. Inhibition of serum lipids was minimal after 1 week of treatment with 4-APP (data not shown) but reached $\sim 60 \%$ after $3-4$ weeks of treatment (Figs. 4 and 5). There may be several reasons for the slow onset of pharmacology. For example, 4-APP (or related metabolites) may accumulate in the liver over time. To test this possibility, we monitored 4-APP levels after 1 week or 4 weeks of oral dosing of 4-APP. Interestingly, 4-APP was not detectable in either serum or liver even after 4 weeks of treatment (when the limit of quantitation was $55 \mathrm{nM}$ ). We hypothesize that 4-APP may be rapidly converted to APPribosides or other nucleic acid analogs as previously reported for orally administered 4-APP in rodents (Henderson and Junga, 1961). It is possible that the 4-APP generated from LY2584702 metabolism may have a different pharmacokinetic profile, leading to apparently higher liver exposure at the single fourth-hour time point following administration of LY2584702 (Fig. 3B).

In an effort to understand which lipid metabolism pathway(s) may be affected by these agents, we carried out an oral fat tolerance test and a VLDL secretion assay following tyloxapol infusion. The data suggest that 3 weeks of oral administration of LY2584702 and low-dose 4-APP had no measurable effect on chylomicron-TG excursion (Fig. 5, B and C). Only modest inhibitory effects on VLDL-TG secretion were observed in the VLDL secretion assay following 4 weeks of treatment (Fig. 5, E and F). Consistent with the modest inhibition of VLDL secretion, we did observe a small increase in liver TG and CE in both LY2584702 and 4-APPtreated groups after 4 weeks of treatment (Fig. 6, A and B). In comparison, treatment with MTPi in LE-DIO rats led to significantly greater accumulation of TG and CE in the liver (Fig. 6, A and B), consistent with the substantial inhibition of VLDL secretion observed with MTPi treatment (Fig. 5, E and F). Lipidomics analysis further revealed $\sim 2$-fold increases in total PE and LPE in liver samples of LY2584702 and 4-APP treatment groups but not in the MTPi treatment group (Fig. 6B). The mechanism for these increases is currently unclear. $\mathrm{PE}$ is a major component of plasma membrane and an important constituent of lipoprotein particles. In liver, high levels of $\mathrm{PE}$ and low $\mathrm{PC} / \mathrm{PE}$ ratios have been associated with risk for steatosis (Arendt et al., 2013). However, in skeletal muscle, total levels of PE are positively associated with insulin sensitivity (Newsom et al., 2016). Whether the increases in PE and LPE associated with LY2584702 and 4-APP may influence lipoprotein metabolism is an interesting topic for future investigation.
One limitation of this work was that no direct assessment of LDL clearance rate occurred in vivo after administration of 4-APP or LY2584702 in LE-DIO rats. However, since serum levels of LDL are balanced by production and clearance, the lack of effect of these agents on chylomicron secretion and their minimal effects on VLDL secretion would suggest that enhanced LDL clearance plays an important role in the robust LDL lowering observed with both 4-APP and LY2584702. In direct support of this notion, we observed a statistically significant increase in LDL uptake after 24 hours of treatment with low concentrations of 4-APP in an in vitro LDL uptake assay in HepG2 cells (Fig. 7A). These activities were not seen with LY2584702 treatment. It is known that HepG2 cells are deficient in oxidative drug metabolism enzymes compared with primary hepatocytes (Wilkening et al., 2003). We reason that during the short treatment duration ( 24 hours) there was insufficient conversion from LY2584702 to 4-APP to produce pharmacology similar to that seen following direct treatment with 4-APP. These data further support the possibility that 4-APP, as a metabolite of LY2584702, may contribute to the in vivo LDL-lowering efficacy following oral administration of LY2584702.

Gene expression data from HepG2 cells and from liver samples of LE-DIO rats suggest that 4-APP can suppress mRNA expression of a panel of lipogenic genes, including ACC1, ACLY, FASN, and SCD1 (Figs. 6C and 7B). These gene expression changes may contribute to the reduction in VLDL secretion and reduction in serum TG observed with 4-APP treatment in vivo (Fig. 5). These lipogenic genes are known to be regulated by transcription factor SREBP1c (Wang et al., 2015), suggesting 4-APP may regulate SREBP1c activity. However, the mechanism by which 4-APP regulates SREBP1c is not yet clear. The p70S6K1 enzyme was previously reported to promote processing and activation of SREBP1c (Owen et al., 2012). However, it is important to note that 4-APP did not demonstrate any measurable p70S6K1 activity in cells (Supplemental Fig. 1A), and thus it must modulate SREBP1c activity via other as yet unknown mechanisms.

4-APP treatment also suppressed PCSK9 and HMGCR mRNA expression levels in both HepG2 cells and in LE-DIO rat liver (Figs. 6C and 7B). Interestingly, LDLR mRNA expression levels were suppressed by 4-APP treatment in LE-DIO rat liver but enhanced in HepG2 cells at high concentrations (Figs. 6C and 7B). This increase in LDLR mRNA expression was also seen in a different human hepatoma cell line Huh7 (data not shown) and in HepG2 cells overexpressing PCSK9 (Supplemental Fig. 2). PCSK9, HMGCR, and LDLR genes are known to be coregulated in similar fashion by transcription factor SREBP2 (Horton et al., 2003). Cholesterol feeding suppresses, whereas cholesterol depletion by statin treatment enhances, mRNA levels for all three genes (Dubuc et al., 2004). This pattern of differential regulation of PCSK9 and LDLR mRNA levels by 4-APP was previously reported with the natural product berberine, which has also been shown to lower LDL in humans (Cameron et al., 2008; Kong et al., 2004). Berberine was reported to increase LDLR mRNA levels through a posttranscriptional mechanism that stabilizes the mRNA (Kong et al., 2004). It is currently unclear whether 4-APP enhances LDLR mRNA levels in human hepatoma cells through a similar mechanism. 
It is important to note that 4-APP treatment in LE-DIO rats significantly lowered LDL-C in the absence of LDLR mRNA increase (Figs. 4C and 6C). This data suggests additional mechanism(s) beyond regulation of LDLR mRNA levels may contribute to the LDL-C-lowering efficacy in vivo. The discrepancy between HepG2 cells and LE-DIO rats in LDLR mRNA regulation may be attributed to differences in compound concentration, treatment duration, species activity, etc. Nevertheless, the phenomenon of 4-APP increasing LDL uptake is in keeping with the in vivo observation that 4-APP significantly lowers LDL-C beyond what can be explained by reduction in VLDL secretion. To our knowledge, this is the first report on hypolipidemic effect of low-dose 4-APP in cells as well as in whole animals. At these low exposure levels, 4-APP was well tolerated with no measureable sign of cytotoxicity. 4-APP itself may not be a suitable agent for treatment of dyslipidemia owing to hepatic lipid accumulation reported in preclinical species as well as coagulopathy reported in humans (Henderson, 1963; Shaw et al., 1960). It would be important to understand whether this mechanism for increasing LDL uptake is independent of 4-APP's other effects. If so, this mechanism could be exploited for future development of LDL-lowering agents that may be complementary to current standard-of-care statins that work through enhancing LDLR mRNA transcription.

In conclusion, we conducted a series of mechanistic studies in preclinical models to investigate potential mechanisms underlying the lipid-lowering efficacy of a p70S6K1 inhibitor LY2584702. The robust LDL-lowering effect observed in humans with this molecule was recapitulated in an LE-DIO rat model. Aside from inhibition of p70S6K1 by LY2584702, we identified an additional mechanism that may contribute to the lipid-lowering efficacy. Specifically, a 4-APP metabolite was found to accumulate in the liver of LE-DIO rats following oral administration of LY2584702. In vivo, treatment with low-dose 4-APP led to reductions in serum LDL-C comparable to LY2584702. In vitro in a human hepatoma HepG2 cell line, 4-APP enhanced LDL uptake. We hypothesize that formation of the 4-APP metabolite contributes to the efficacy of LY2584702 in lowering LDL-C in the LE-DIO rat model. This mechanism may be relevant to the lipidlowering efficacy in humans observed with LY2584702.

\section{Acknowledgments}

The authors thank Dr. John Wetterau for critically reading the manuscript. We thank Dr. David J. DeBrota, Colin F. Green, Dr. Michael Hodsdon, Dr. Sunil K. Kadam, Dr. Sajan Joseph, Dr. Nathan B. Mantlo, Dr. Kenneth D. Roth, and Dr. Jeffrey G. Suico for scientific discussions. We thank Rose Ajamie, David W. Bedwell, Kevin Black, Brenda Hanssen, Krista K. Linville Kenneth J. Ruterbories, Darlene Satonin, Jill Schroeder, John P. Stutz, and Guoming Wang for technical support.

\section{Authorship Contributions}

Participated in research design: Estridge, Dey, Yu, Zhang, Jin, Kowala, Leohr, Fretland, Mabry, Luffer-Atlas, Luo.

Conducted experiments: Estridge, Dey, Reidy, Yu, Zhang, Hartley, Milligan.

Performed data analysis: Estridge, Dey, Reidy, Yu, Zhang, Hartley, Milligan, Fretland, Luffer-Atlas, Luo.

Wrote or contributed to the writing of the manuscript: Estridge, Dey, Reidy, Yu, Zhang, Hartley, Milligan, Jin, Kowala, Leohr, Fretland, Mabry, Luffer-Atlas, Luo.

\section{References}

Arendt BM, Ma DW, Simons B, Noureldin SA, Therapondos G, Guindi M, Sherman M, Allard JP (2013) Nonalcoholic fatty liver disease is associated with lower hepatic and erythrocyte ratios of phosphatidylcholine to phosphatidylethanolamine. Appl Physiol Nutr Metab 38: 334-340

Cameron J, Ranheim T, Kulseth MA, Leren TP, and Berge KE (2008) Berberine decreases PCSK9 expression in HepG2 cells. Atherosclerosis 201:266-273.

Dubuc G, Chamberland A, Wassef H, Davignon J, Seidah NG, Bernier L, and Prat A (2004) Statins upregulate PCSK9, the gene encoding the proprotein convertase neural apoptosis-regulated convertase-1 implicated in familial hypercholesterolemia. Arterioscler Thromb Vasc Biol 24:1454-1459.

Funatsu T, Kakuta H, Takasu T, and Miyata K (2002) Atorvastatin increases hepatic fatty acid beta-oxidation in sucrose-fed rats: comparison with an MTP inhibitor. Eur J Pharmacol 455:161-167.

Goldstein JL and Brown MS (2015) A century of cholesterol and coronaries: from plaques to genes to statins. Cell 161:161-172.

Han B, Eacho PI, Knierman MD, Troutt JS, Konrad RJ, Yu X, and Schroeder KM (2014) Isolation and characterization of the circulating truncated form of PCSK9. $J$ Lipid Res 55:1505-1514.

Henderson JF (1963) Studies on Fatty Liver Induction by 4-Aminopyrazolopyrimidine. J Lipid Res 4:68-74.

Henderson JF and Junga IG (1961) The metabolism of 4-aminopyrazolo(3,4-d)pyrimidine in normal and neoplastic tissues. Cancer Res 21:118-129.

Hollebecque A, Houédé N, Cohen EE, Massard C, Italiano A, Westwood P, Bumgardner W, Miller J, Brail LH, Benhadji KA, et al. (2014) A phase Ib trial of LY2584702 tosylate, a p70 S6 inhibitor, in combination with erlotinib or everolimus in patients with solid tumours. Eur J Cancer 50:876-884.

Horton JD, Cohen JC, and Hobbs HH (2009) PCSK9: a convertase that coordinates LDL catabolism. J Lipid Res 50 (Suppl):S172-S177.

Horton JD, Shah NA, Warrington JA, Anderson NN, Park SW, Brown MS, and Goldstein JL (2003) Combined analysis of oligonucleotide microarray data from transgenic and knockout mice identifies direct SREBP target genes. Proc Natl Acad Sci USA 100:12027-12032.

Kieft KA, Bocan TM, and Krause BR (1991) Rapid on-line determination of cholesterol distribution among plasma lipoproteins after high-performance gel filtration chromatography. J Lipid Res 32:859-866.

Kong W, Wei J, Abidi P, Lin M, Inaba S, Li C, Wang Y, Wang Z, Si S, Pan H, et al. (2004) Berberine is a novel cholesterol-lowering drug working through a unique mechanism distinct from statins. Nat Med 10:1344-1351.

Krause BR, Sloop CH, and Roheim PS (1981) Lipid absorption in unanesthetized, unrestrained rats. Effects of 4-aminopyrazolopyrimidine and ethinyl estradiol. Biochim Biophys Acta 665:165-169.

Li S, Ogawa W, Emi A, Hayashi K, Senga Y, Nomura K, Hara K, Yu D, and Kasuga M (2011) Role of S6K1 in regulation of SREBP1c expression in the liver. Biochem Biophys Res Commun 412:197-202.

Li S, Zhang HY, Hu CC, Lawrence F, Gallagher KE, Surapaneni A, Estrem ST, Calley JN, Varga G, Dow ER, et al. (2008) Assessment of diet-induced obese rats as an obesity model by comparative functional genomics. Obesity (Silver Spring) 16: 811-818.

Magnuson B, Ekim B, and Fingar DC (2012) Regulation and function of ribosomal protein S6 kinase (S6K) within mTOR signalling networks. Biochem J 441:1-21.

Newsom SA, Brozinick JT, Kiseljak-Vassiliades K, Strauss AN, Bacon SD, Kerege AA, Bui HH, Sanders P, Siddall P, Wei T, et al. (2016) Skeletal muscle phosphatidylcholine and phosphatidylethanolamine are related to insulin sensitivity and respond to acute exercise in humans. J Appl Physiol 120: 1355-1363

Owen JL, Zhang Y, Bae SH, Farooqi MS, Liang G, Hammer RE, Goldstein JL, and Brown MS (2012) Insulin stimulation of SREBP-1c processing in transgenic rat hepatocytes requires p70 S6-kinase. Proc Natl Acad Sci USA 109: 16184-16189.

Pearce LR, Komander D, and Alessi DR (2010) The nuts and bolts of AGC protein kinases. Nat Rev Mol Cell Biol 11:9-22.

Rader DJ and Kastelein JJ (2014) Lomitapide and mipomersen: two first-in-class drugs for reducing low-density lipoprotein cholesterol in patients with homozygous familial hypercholesterolemia. Circulation 129:1022-1032.

Ricoult SJ and Manning BD (2013) The multifaceted role of mTORC1 in the control of lipid metabolism. EMBO Rep 14:242-251.

Seidah NG, Awan Z, Chrétien M, and Mbikay M (2014) PCSK9: a key modulator of cardiovascular health. Circ Res 114:1022-1036.

Shaw RK, Shulman RN, Davidson JD, Rall DP, and Frei, 3rdE (1960) Studies with the experimental antitumor agent 4-aminopyrazolo[3, 4-d]pyrimidine. Cancer 13: 482-489.

Shiff TS, Roheim PS, and Eder HA (1971) Effects of high sucrose diets and 4 -aminopyrazolopyrimidine on serum lipids and lipoproteins in the rat. J Lipid Res 12:596-603.

Tolcher A, Goldman J, Patnaik A, Papadopoulos KP, Westwood P, Kelly CS, Bumgardner W, Sams L, Geeganage S, Wang T, et al. (2014) A phase I trial of LY2584702 tosylate, a p70 S6 kinase inhibitor, in patients with advanced solid tumours. Eur J Cancer 50: 867-875.

Wang Y, Viscarra J, Kim SJ, and Sul HS (2015) Transcriptional regulation of hepatic lipogenesis. Nat Rev Mol Cell Biol 16:678-689.

Wilkening S, Stahl F, and Bader A (2003) Comparison of primary human hepatocytes and hepatoma cell line Hepg2 with regard to their biotransformation properties. Drug Metab Dispos 31:1035-1042.

Address correspondence to: Dr. M. Jane Luo, CardioMet Disease/Diabetic Complications, Eli Lilly \& Co., Lilly Corporate Center, Indianapolis, IN, 46285. E-mail: luomi@lilly.com 